\title{
Carta 2011
}

Ruth Amstein, Jan Steffel, Thomas F. Lüscher

Zurich Heart House -

Foundation for Cardiovascular Research, Zurich und Klinik für Kardiologie, HerzKreislaufZentrum, UniversitätsSpital Zürich
Korrespondenz:

Dr. Ruth Amstein

Zurich Heart House -

Foundation for Cardiovascular

Research

Moussonstrasse 4

CH-8091 Zürich

ruth.amstein[at]usz.ch

\section{Öffentliche Forschungsfinanzierung muss besser werden}

Prof. Thomas Lüscher eröffnete die Veranstaltung mit einer kurzen Übersicht. In der Spitzenmedizin, so Lüscher, komme es vor allem auf Personen an, welche diese durch ihren Pioniergeist und ihre herausragenden individuellen Leistungen prägen. Eine wichtige Voraussetzung dafür sei auch die Forschung. Die Schweiz stehe im internationalen Vergleich gut da: Im neuesten Ranking der europäischen Hochschulen sind sowohl die ETH Zürich, die EPFL Lausanne sowie die Universität Zürich unter den besten 25 Hochschulen vertreten [1]. Neben Persönlichkeiten seien auch Strukturen entscheidend, um die Entfaltung dieser Personen und ihrer Teams optimal zu ermöglichen; hier sieht er vor allem die Politik gefordert, solche Voraussetzungen zu schaffen. Das schliesse insbesondere auch die Finanzierung mit ein: In der Schweiz werde gegenwärtig nämlich die Forschung zu zwei Dritteln über private Geldgeber finanziert [2], während sie in anderen Ländern wie beispielsweise Deutschland zu einem höheren Anteil vom Staat durch Exzellenzinitiativen [3] und Netzwerke unterstützt werde. Daneben sei aber auch eine Re-Evaluation der Rolle der Industrie erforderlich, deren Unterstützung häufig in ein negatives, mit Interessenkonflikten behaftetes Licht gerückt werde. Im Gegensatz, so Lüscher, seien Industrie und Wirtschaft nicht nur als wichtige Geldgeber, sondern auch bei der Umsetzung im translationalen Forschungsbereich (from bench to bedside) entscheidende Partner von akademischen Forschenden. Darüber hinaus müssten auch karitative Geldgeber über entsprechende Anreize gefördert werden, wie dies beispielsweise in den USA der Fall sei [4].

\section{Bedeutung der Spitzenmedizin für die Schweiz}

Ständerat Prof. Felix Gutzwiller betonte, dass die Ausgaben für die Spitzenmedizin mit $4 \%$ des Gesundheitssystems absolut gesehen einen eher kleinen Anteil ausmachten. In der Politik werde das Gesundheitssystem zu häufig lediglich als Kostenfaktor angesehen, während positive Aspekte wie der Nutzen im Beschäftigungs- und Innovationssektor ignoriert würden. Darüber hinaus profitiere auch der Industriestandort Schweiz mit dem Pharma- und Medizinaltechniksektor von einem starken Gesundheitssystem. Entsprechend sei die Stagnation der Investitionen in die Forschung nicht nachvollziehbar, während gleichzeitig an-

\section{Carta 2011}

Le 21 octobre 2011 s'est tenue à l'hôpital universitaire de Zurich la huitième «Cardiovascular Roundtable» (CARTA). Cette manifestation a pour objectif de réunir les responsables de l'industrie technique médicale et pharmaceutique, le corps médical, les assureurs-maladie et les politiciens, et d'instaurer un dialogue ouvert entre les différents partenaires de la santé. La CARTA 2011, présidée par les professeurs Felix H. Sennhauser et Thomas F. Lüscher, était consacrée à la place de la médecine de pointe en Suisse. Si celle-ci dépend principalement de personnalités douées d'un esprit d'initiative hors du commun et capables de motiver toute une équipe, la recherche et l'encouragement de la relève sont également indispensables. Il convient aussi d'assurer à ces équipes un environnement et des structures favorables devant permettre leur épanouissement, et d'amener le milieu politique à financer davantage le secteur de la recherche et du développement, car la médecine de pointe joue un rôle économique non négligeable pour la Suisse, également en tant que bien d'exportation.

dere Länder ihre Forschungsgelder anheben. Hiermit könnte in naher Zukunft ein Nachteil für die Schweiz entstehen, da künftige Wertschöpfungen entscheidend von heutigen Investitionen mitbestimmt würden. Ausserdem müsse die Forschung, so Gutzwiller, unter den Schweizer Zentren besser koordiniert werden, um Redundanz und Überschneidungen zu vermeiden und Synergien zu fördern. Gleichzeitig müsse eine Überregulierung vermieden werden, um die Forschungsfreiheit nicht einzuschränken und Individualität zu bewahren. Als positives Beispiel nannte Gutzwiller in diesem Zusammenhang die zukünftige Koordination der Ethikkommissionen.

Die in Kürze zu verabschiedende Botschaft zu Bildung, Forschung und Innovation (BFI) des Bundesrates wird für die Nachhaltigkeit der Förderung der Spitzenmedizin entscheidend sein. Für den Zeitraum 


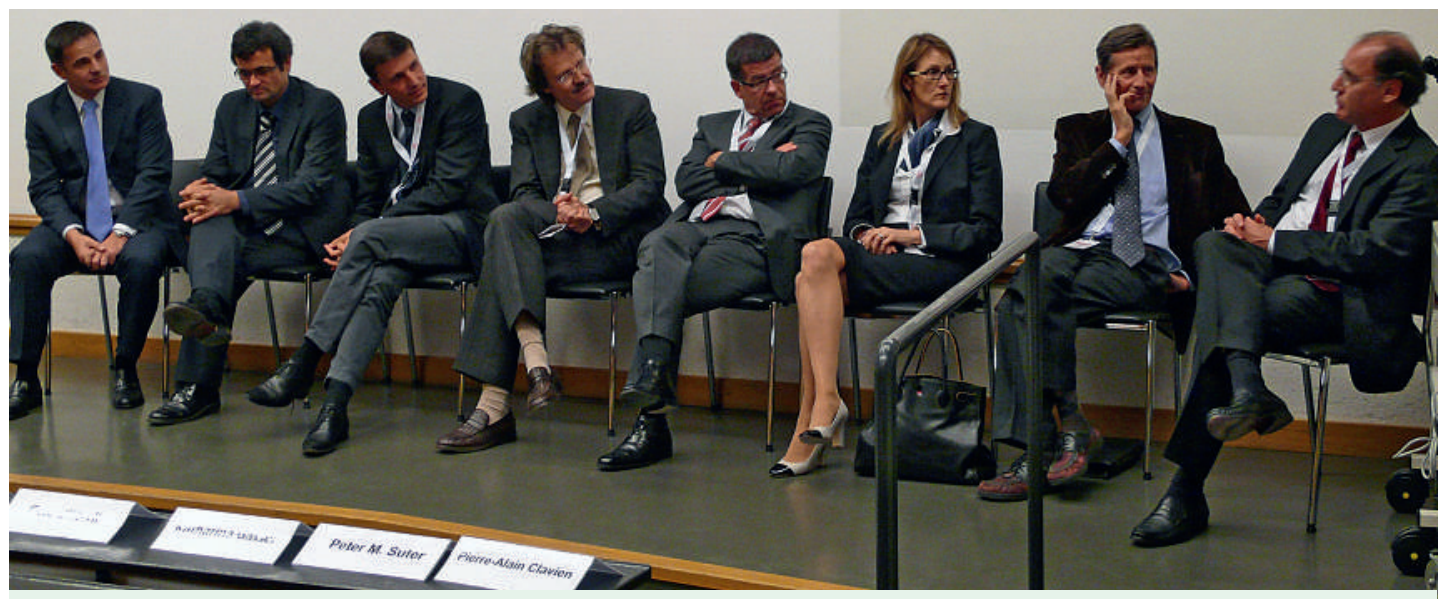

Podiumsdiskussion zwischen den Partnern des Gesundheitswesens. V.I.n. r.: Albert Panzeri (Biotronik), Rudolf Minsch (Economiesuisse), Franz Immer (Swisstransplant), Thomas F. Lüscher (UniversitätsSpital Zürich), Otto Bitterli (Sanitas), Katharina Gasser (Novartis), Peter Suter (Fachorgan hochspezialisierte Medizin), Pierre-Alain Clavien (UniversitätsSpital Zürich). Foto Hans Utzinger, USZ.

2013-2016 werde ein Wachstumsziel von 6\% angestrebt; dabei soll die Exzellenzförderung und -finanzierung besondere Aufmerksamkeit erfahren. Unglücklicherweise werde der Spitzenmedizin im Parlament nicht immer die Bedeutung beigemessen, die sie verdiene, da sie im Gegensatz beispielsweise zur Landwirtschaft fast keine Interessenvertreter habe.

\section{Spitzenmedizin ist Teamarbeit mit einem Leader}

Prof. Peter Suter sieht bei der Spitzenmedizin die Qualität der Leistungen im Vordergrund; diese umfassen das ganze Spektrum vom Kunstherz bis zur Palliativmedizin und sollten prinzipiell der gesamten Bevölkerung zugänglich sein. Die Spitzenmedizin orientiere sich klar an der Evidenz-basierten Medizin als Garant für Qualität, Wirksamkeit und Kosteneffizienz. Transparenz für Resultate, aber auch für Komplikationen sei darüber hinaus wichtig, die zum Beispiel in Form von Registern erfasst werden können. Spitzenmedizin sei ganz klar Teamarbeit mit einem Leader. Es brauche hierbei Mut zu Entwicklung, Innovation und Forschung. In speziellen Netzwerken, sowohl mit der Industrie (in der Entwicklung), als auch mit Hausärzten (in der Implementierung und damit der Versorgung) könne dann die Spitzenmedizin im Sinne einer klassischen «Win-win-Situation» für alle Teile der Gesellschaft praktiziert werden.

\section{Zertifizierung für die Spitzenmedizin gefordert}

Für Prof. Pierre-Alain Clavien sind die Voraussetzungen für die Spitzenmedizin in der Schweiz insgesamt nicht schlecht. Das Hauptproblem der Spitzenmedizin, so Clavien, sei die kritische Masse an Eingriffen, die es benötigt, um selbige auch mit der nötigen Qualität und wissenschaftlichem Impact umsetzen zu können. Entsprechend sei eine Zentralisierung auf wenige, hochqualifizierte Zentren sowohl medizinisch als auch volkswirtschaftlich eine unabdingbare conditio sine qua non: je höher das Volumen, desto besser die Ergebnisse (als Beispiel führt er die Leber- und Ösophaguschirurgie an) und desto geringer die Kosten. In der Schweiz sind hierfür die Voraussetzungen mit den Listenspitälern geschaffen worden, die eine minimale Fallzahl für bestimmte Eingriffe vorsehen. Ausserdem sei eine Verbesserung der interkantonalen Koordination ebenso vonnöten wie eine Zertifizierung von Ärzten und Zentren, die hochspezialisierte Eingriffe durchführen. Clavien beklagte die enorme Zunahme der Bürokratie sowie die fehlende Involvierung akademischer Leiter in die Entscheidungsprozesse medizinischer Institutionen. Schliesslich sei auch die Einführung der 50-Stunden-Woche zur Ausbildung von Spitzenmedizinern kontraproduktiv, da hiermit nicht nur dem Ausbildner, sondern auch den Auszubildenden Limitationen auferlegt würden, die eine klinische und wissenschaftliche Ausbildung im Bereich Spitzenmedizin unmöglich machten.

\section{Wirtschaftliche Bedeutung der Spitzenmedizin}

Die Spitzenmedizin spielt laut Prof. Rudolf Minsch wirtschaftlich eine gewichtige Rolle. Im Gesundheitswesen seien folgende Trends zu beobachten: Bei steigender Lebenserwartung wachsen die Gesundheitskosten im Vergleich zum Bruttoinlandprodukt überproportional an. Um den steigenden Kosten entgegenzutreten, sei über die letzten Jahre eine schleichende Verstaatlichung des Gesundheitswesens zu beobachten.

Die Spitzenmedizin stelle aus ökonomischer Sicht ein natürliches Monopol dar: Da die Kosten pro Patient primär hoch sind, bei steigender Anzahl an Behandlungen jedoch fallen, wäre in der Spitzenmedizin formal ein einziges Zentrum pro hochspezialisierten Eingriff ökonomisch und medizinisch gesehen 
ausreichend. Spitzenmedizin sei jedoch auch ein dynamischer Prozess, da Eingriffe mit der Zeit zur klinischen Routine werden - mit entsprechender Ausweitung der Indikationsstellung, der beteiligten Zentren und in der Folge der Fallzahlen. Spitzenmedizin brauche daher sowohl eine vorausschauende Planung als auch Innovationsgeist. Spitzenmedizin könne nur durch Innovation Spitze sein, meinte Minsch, und es brauche dafür ausreichend öffentliche Mittel. Gute Innovationspolitik sei allerdings schwierig zu betreiben und könne nicht mit planwirtschaftlichen Ansätzen gesteuert werden. Minsch sieht hier aber einen lohnenden Investitionssektor mit nachhaltiger Wirkung auch für die wichtigen Exportbranchen wie die Pharma- und Medizinaltechnikindustrie. Die Konkurrenz im Ausland dürfe dabei nicht unterschätzt werden, aber die Schweiz sei innovativ genug, um in einzelnen Disziplinen die Weltspitze anzuführen.

\section{Forschung und Nachwuchsförderung - zwingend für die Spitzenmedizin?}

Forschung und Nachwuchsförderung sind zwingende, integrale Bestandteile der Spitzenmedizin, so die Quintessenz der ersten Breakout-Session, die von Prof. Simon Hoerstrup und Prof. Felix Sennhauser geleitet und zusammengefasst wurde. Die Nachwuchsförderung habe dabei insbesondere zum Ziel, eine sich abzeichnende Abhängigkeit von ausländischem Influx von Akademikern zu vermeiden. Hierzu müssten nicht nur Persönlichkeiten und Talente früh entdeckt und systematisch gefördert, sondern auch entsprechende Ressourcen zur Verfügung gestellt werden. Entsprechende Strukturen wie die Begabtenförderung, die MD-PhD-Programme sowie die Förderungsprofessuren des Schweizerischen Nationalfonds seien Schritte in die richtige Richtung. Dies sei umso wichtiger, da die Finanzierung aus der Industrie aufgrund von DRGs und Fallpauschalen wahrscheinlich abnehmen werde.

\section{Bringt Spitzenmedizin internationale Exzellenz?}

Prof. Ralph Müller und Prof. Gustav von Schulthess stellten in der Zusammenfassung der zweiten Breakout-Session zunächst fest, dass der Begriff «Spitzenmedizin» eher unglücklich gewählt und die Formulierung «Hochspezialisierte Medizin» deutlich passender und auch im Englischen verwendbar wäre. Zweifelsohne können durch die Förderung der hochspezialisierten Medizin auch die Voraussetzungen für internationale Exzellenz geschaffen werden. Im Umkehrschluss sei es sogar entscheidender, sich vor allem im internationalen Umfeld zu messen, als lokale Mitstreiter als Massstab zu nehmen. Ein wichtiger Diskussionspunkt dieser Arbeitsgruppe war die Frage, wieso in erster Linie angelsächsische Universitäten weltweit seit langem die Spitzengruppe anführen. Hier gäbe es einiges zu lernen, so insbesondere die Auswahl der Nachwuchstalente, das motivierende Teaching, die hohe Stellung von Exzellenz und akademischem Unternehmertum sowie die wichtige Rolle der Charity zur Finanzierung von Grossprojekten.

\section{Was darf Spitzenmedizin kosten?}

In der dritten Breakout-Session wurde der Kostenaspekt der Spitzenmedizin durch Otto Bitterli, Mayella Favre und Dr. Ruth Amstein diskutiert. Für die Frage der Kostenübernahme sind Qualitätssicherung und die Schaffung von Transparenz wichtig. Da die Leistungen der Spitzenmedizin mit 4\% nur einen kleinen Teil der gesamten Gesundheitskosten ausmachen, sei das Problem für die Zusatzversicherung gering. Von der Grundversicherung können jedoch nur solche Leistungen übernommen werden, die im Leistungskatalog des KVG aufgeführt sind. Für etablierte spitzenmedizinische Leistungen ist dies in vielen Fällen bereits Realität. Viele Leistungen der Spitzenmedizin fallen aber nicht zwingend hierunter, da es sich oft um hochmoderne, neue und teilweise noch nicht vollständig etablierte Prozeduren handelt. Eine Mischfinanzierung durch Versicherungen, Staat und Industrie (Letztere insbesondere in der Forschung, Entwicklung und Anfangsphase der klinischen Einführung neuer Geräte und Eingriffe) wäre ein gangbarer Weg.

In der anschliessenden Podiumsdiskussion wurden die Themen noch einmal zusammenfassend behandelt. Otto Bitterli wies darauf hin, dass die Finanzierung gerade für neue Eingriffe, die noch nicht offiziell eingeführt sind, an einem runden Tisch von Versicherern und Universitätsspitälern zu besprechen sei, ein Vorschlag, der von allen positiv aufgenommen wurde. Albert Panzeri betonte vonseiten der Gerätehersteller, dass die Zusammenarbeit mit Schweizer Spitzenspitälern für die forschende Industrie essentiell sei, dass diese aber nur zu einem Preis geschehen könne, der auch die Kosten decke. Das Problem stelle sich, wie Dr. Katharina Gasser ausführte, in der Pharmaindustrie in einem geringeren Ausmass, aber auch sie seien einem starken Kostendruck ausgesetzt, würden aber weiterhin gerne mit Schweizer Zentren ihre Produkte entwickeln. PierreAlain Clavien betonte in seinem Schlusswort, dass bei allen Problemen, die die Schweizer Spitzenmedizin zu bewältigen habe, die Schweiz ein hohes Potential besitze, auch weiterhin international eine wichtige Rolle zu spielen, wenn sich die Universitäten auf ihre Stärken konzentrierten und dort ihre Mittel präferentiell einsetzen würden.

\section{Referenzen}

1 Thomson Reuters. The World University Rankings. www.timeshighereducation.co.uk

2 Bundesamt für Statistik. BFS. letzter Stand 2008.

3 Exzellenz-Initiative Deutschland: www.exzellenz-initiative.de

4 Lüscher TF, Amstein R. Charity in Gesellschaft und Wissenschaft - Andrew Carnegie revisited. Cardiovasc Med. 2011;14(9):235-41. 